umständliche (vgl. etwa S. 59f.) Untersuchung der bei Hobbes konstatierten Metaethik tatsächlich ein Zugewinn für das Verständnis von Hobbes bedeutet. Letztlich muss Abizadeh doch zugestehen, dass ,,much of this set of metaethical commitments is implicit rather than fully worked out in Hobbes“ (S. 276).

\title{
Scattola, Merio: Prinzip und Prinzipienfrage in der Entwicklung des modernen Naturrechts, 283 S., frommann-holzboog, Stuttgart-Bad Cannstatt 2018.
}

\author{
Peter Nitschke \\ Online publiziert: 19. Februar 2020 \\ (C) Der/die Autor(en) 2020
}

Merio Scattola ist in Deutschland gewiss kein Unbekannter. Dennoch ist der viel zu früh (2015) verstorbene Ideenhistoriker aus Padua meist nur einem kleineren Kreis von Experten in der Rechts- und Ideengeschichte oder der Politischen Philosophie bekannt. Das liegt an seinem Forschungsschwerpunkt, in dem er sich immer wieder und intensiv mit Fragestellungen der Genese des modernen Naturrechts vom 16. bis zum 18. Jahrhundert beschäftigt hat. Im Gegensatz zu vielen anderen ist Scattola hierbei besonders gern auf die lateinischen Originalfassungen der Publikationen sei es aus der Schule von Salamanca, sei es anhand der überaus zahlreichen Schriften protestantischer Gelehrter des 17. Jahrhunderts - eingegangen. In der HerzogAugust-Bibliothek von Wolfenbüttel und am Max-Planck-Institut für europäische Rechtsgeschichte in Frankfurt war er daher oft und gern gesehener Gast.

Akribisch und subtil hat Scattola hier seine Studien an schwierigen Texten zur Entstehung nicht nur des naturrechtlichen, sondern, darin verwoben, auch des modernen politischen Denkens erarbeitet und im deutschsprachigen wie italienischen Raum, zuletzt zunehmend auch im englischen Format, vorgestellt. Seine Interpretationen und Analysen kommen nicht wortgewaltig im Sinne einer Effekthascherei daher. Vielmehr sind sie um die jeweils sachlich konkrete Rekonstruktion der zu verhandelnden Topoi bemüht, und versuchen diese einzuordnen in einen Diskurszusammenhang, der verschiedene Autoren der Prämoderne inhaltlich miteinander verbindet oder eben auch strukturell unterscheidet. Wie auch bei der Cambridge

P. Nitschke $(\triangle)$

Universität Vechta, Vechta, Deutschland

E-Mail: peter.nitschke@uni-vechta.de 
School geht es Scattola um eine Kontextualisierung des jeweiligen Diskurses, sei es zur Frage des ius naturae, zur Frage der Politik oder der Verbindung von Moral, Religion und Politik im naturrechtlichen Denken der Prämoderne. Anders jedoch als bei den maßgeblichen Vertretern der Cambridge School zielt die Argumentation bei Scattola zunächst immer darauf, den Text selbst richtig verstehen zu können und ihn dann einzuordnen in den Interpretationsstrom nachfolgender oder zeitgleich lebender Autoren. Es ist hierbei nicht so sehr das Pro und Kontra im Diskurs, etwa das Antwortecho auf bestimmte Theoreme, sondern die Stringenz einer systemischen oder durchaus metaphysischen Konstellation in den Texten zum Naturrecht, an dem ihm hierbei liegt. Die zum Teil scharfen Frontstellungen der Cambridge School sind daher auch nicht seine Sache. Interpretatorisch neigt Scattola dazu, die Autoren mit den eigenen Aussagen in längeren Zitaten zu Wort kommen zu lassen. Das wirkt oft so, als habe man es mit einer historischen Quelle zu tun, die mitunter allzu deskriptiv vorgetragen wird. Da es sich um lateinische oder italienische Texte handelt, werden sie immerhin im Argumentationsteil wie in den Fußnoten in deutscher Übersetzung von Scattola dokumentiert.

Der vorliegende Band beinhaltet eine Zusammenstellung verschiedener Beiträge von Scattola, an deren redaktioneller Bearbeitung er noch bis kurz vor seinem Tode selbst mitgewirkt hat. Der Herausgeber Andreas Wagner hat hierzu ein sehr schönes und informatives Vorwort geschrieben. Allerdings wird nicht dokumentiert, in welchem zeitlichen und inhaltlichen Kontext diese Beiträge zustande gekommen sind. Bis auf einen Aufsatz („Principium oder principia?“) sind die sechs übrigen Beiträge offenbar vorher noch nicht publiziert worden, jedenfalls gibt es dazu keinen editorischen Hinweis.

Ausgehend von Hugo Grotius als dem gemeinhin als Schöpfer des modernen Naturrechts angesehenen Autor beschäftigt sich Scattola in den hier dokumentierten Beiträgen zentral mit der Frage nach der Relation von Inhalt (dem Naturrecht) und der Methodik seiner Begründung. Der Titel des Buches ist insofern tatsächlich programmatisch: es geht um die Prinzipien in der Begründung des neuen Naturrechts, das sich vom alten (klassischen), dem christlich-mittelalterlichen ius naturae abhebt beziehungsweise herauskristallisiert. Doch so schematisch, wie in den heutigen Lehrbüchern meist angenommen, ist dieser Vorgang keineswegs. Die Argumente zugunsten einer Säkularisierung und damit Individualisierung des menschlichen Subjekts verlaufen nicht geradlinig, sondern gehen hin und her, selbst bei Grotius, der tief religiös ist. Die Frage ist eher, und darauf weist Scattola zu Recht hin, ob nicht im Rahmen einer Betrachtung der innerweltlichen Problemlagen, insbesondere im Bereich der Politik, eine andere Auffassung von Wahrheit oder Realität angesetzt werden muss als in der reinen Lehre der Gottesbetrachtung? Das Ergebnis ist historisch bekannt, doch Scattolas Beiträge in diesem Band zeichnen den verschlungenen und letztlich gar nicht so einheitlich kodierten Weg über die Herstellung von Historizität, die Implementierung machiavellischer Theoreme in die Naturrechtsdebatte und die Transformationen der juridischen Begründung im 17. Jahrhundert sehr schön nach. In Auseinandersetzung mit Samuel von Pufendorf kann Scattola alsdann zeigen, wie sehr sich die Debatte im Verlauf des 18. Jahrhunderts, beispielhaft mit der Argumentation der beiden Göttinger Rechtsgelehrten Johann Jakob Schmauß (1690-1757) und Johann Christian Claproth (1715-1748), vom rationa- 
lisierenden Intellektualismus gelöst hat und neue Wege hin zu einer sensitiven, handlungstheoretischen Argumentation gegangen ist. Scattola wertet dies sogar als erste Anzeichen für die Generierung eines sozialwissenschaftlichen Verständnisses. Abgeschlossen wird die Perspektive mit einem überaus lesenswerten Beitrag über die Philosophie des Naturrechts, wie sie Luigi Taparelli d'Azeglio (1793-1862) in Italien formuliert hat. Wenn man so will, stellt das den metaphysischen Gegenpart zur vorherigen Argumentation dar. Denn in dieser sehr katholischen Auslegung zum Naturrecht kommt Gott zurück ins Zentrum der Auseinandersetzungen, dieses Mal jedoch (wieder) hinsichtlich der Möglichkeiten der menschlichen Vernunft, die ihrerseits nur das erkennen kann, was Gott in der Natur gesetzt hat. Auch wenn damit eine thomistische Ordo-Position zurückkehrt in den Diskurs, bildet dieses Kapitel einen gelungenen Abschluss für das thematische Spektrum des ganzen Bandes, der mit einer Vielzahl von Textpartien bekannter und weniger bekannter Autoren aufwartet.

Funding Open Access funding provided by Projekt DEAL.

Open Access Dieser Artikel wird unter der Creative Commons Namensnennung 4.0 International Lizenz veröffentlicht, welche die Nutzung, Vervielfältigung, Bearbeitung, Verbreitung und Wiedergabe in jeglichem Medium und Format erlaubt, sofern Sie den/die ursprünglichen Autor(en) und die Quelle ordnungsgemäß nennen, einen Link zur Creative Commons Lizenz beifügen und angeben, ob Änderungen vorgenommen wurden.

Die in diesem Artikel enthaltenen Bilder und sonstiges Drittmaterial unterliegen ebenfalls der genannten Creative Commons Lizenz, sofern sich aus der Abbildungslegende nichts anderes ergibt. Sofern das betreffende Material nicht unter der genannten Creative Commons Lizenz steht und die betreffende Handlung nicht nach gesetzlichen Vorschriften erlaubt ist, ist für die oben aufgeführten Weiterverwendungen des Materials die Einwilligung des jeweiligen Rechteinhabers einzuholen.

Weitere Details zur Lizenz entnehmen Sie bitte der Lizenzinformation auf http://creativecommons.org/ licenses/by/4.0/deed.de. 